\title{
Quality of life in patients with Parkinson's disease: development of a questionnaire
}

\author{
A G E M de Boer, W Wiiker, J D Speelman, J C J M de Haes
}

\begin{abstract}
Objectives-To develop and test a questionnaire for measuring quality of life in patients with Parkinson's disease.

Methods-An item pool was developed based on the experience of patients with Parkinson's disease and of neurologists; medical literature on the problems of patients with Parkinson's disease; and other quality of life questionnaires. To reduce the item pool, 13 patients identified items that were a problem to them and rated their importance. Items which were most often chosen and rated most important were included in the Parkinson's disease quality of life questionnaire (PDQL). The PDQL consists of 37 items. To evaluate the discriminant validity of the PDQL three groups of severity of disease were compared. To test for convergent validity, the scores of the PDQL were tested for correlation with standard indices of quality of life.
\end{abstract}

Results-The PDQL was filled out by 384 patients with Parkinson's disease. It consisted of four subscales: parkinsonian symptoms, systemic symptoms, emotional functioning, and social functioning. The internal-consistency reliability coefficients of the PDQL subscales were high $(0 \cdot 80-0 \cdot 87)$. Patients with higher disease severity had significantly lower quality of life on all $P D Q L$ subscales $(P<0.05)$. Almost all PDQL subscales correlated highly $(P<0.001)$ with the corresponding scales of the standard quality of life indices.

Conclusion-The PDQL is a relevant, reliable, and valid measure of the quality of life of patients with Parkinson's disease.

$(\mathfrak{F}$ Neurol Neurosurg Psychiatry 1996;61:70-74)

Medical Psychology

A G E M de Boer

W Wijker

J C J M de Haes

Department of

Neurology, University

of Amsterdam,

Amsterdam, The

Netherlands

J D Speelman

Correspondence to:

Dr A G E M de Boer,

Dreartment of Medic

Department of Medica

Medical Centre, University

Medical Centre, University

of Amsterdam, Meibergdre

The Netherlands.

Received 6 October 1995

and in revised form

12 March 1996

Accepted 12 March 1996

Keywords: quality of life; Parkinson's disease; questionnaire development

Traditionally, the primary focus of medical research is the measurement of mortality and morbidity. During the past decade however, interest is increasing in measurement of quality of life, health status, and functional status. Particularly in the field of chronic disease, it has been argued that greater attention should be paid to the impact of illness and treatment on the functional, emotional, and social wellbeing of a patient.
Parkinson's disease has a major adverse impact on patient's lives. Patients not only experience functional impairment, but the disease may also affect their emotional and social life. ${ }^{1-3}$ There are some instruments available that measure severity of disease, ${ }^{4-7}$ but these do not focus on the patient's subjective experience of the illness. Moreover, most of these indices are given by physicians and not by patients and none of these instruments includes the emotional and social domains of health. Other generic quality of life instruments aim at assessing the quality of life of general populations and do not concentrate on the specific problems of patients with Parkinson's disease. ${ }^{8-10}$

The purpose of this study was to develop a disease specific instrument to measure quality of life of patients with Parkinson's disease, and to test the internal-consistency reliability and its validity.

\section{Methods}

PRINCIPLES OF DISEASE SPECIFIC

QUESTIONNAIRE DEVELOPMENT

Guyatt, Kirshner, Juniper, et al have described criteria that should be met in questionnaire development. ${ }^{11-14}$ These criteria are:

- Items must reflect areas of function that are important to patients with Parkinson's disease

- Both physical and emotional health should be measured

- Summary scores should be amenable to statistical analysis

- The questionnaire should be valid

- The questionnaire should be relatively short and simple.

We have developed the questionnaire based on these criteria. Firstly, to ensure that the questionnaire is relevant, an item pool was selected and then reduced. Secondly, we tested the questionnaire for reliability and validity. The study design was approved by the hospital ethics committee.

\section{PHASE 1: ITEM SELECTION AND REDUCTION}

First of all we wanted to identify items of quality of life impairment that might be relevant for patients with Parkinson's disease. An open patients recruited from the university clinic. Based on these interviews an item pool (the reduction questionnaire) was constructed. Other items were added to the item reduction questionnaire, based on the personal experience of neurologists specialised in Parkinson's disease $(n=3)$; the personal experience of the interview was conducted with four consecutive 
board of the Dutch Parkinson's Disease Society (a patient and a relative, $\mathrm{n}=2$ ); a review of the medical literature concerning the problems of patients with Parkinson's disease; and other disease specific instruments. ${ }^{11} 1314$

Once this item reduction questionnaire had been generated, 13 consecutive patients of the outpatients' and inpatients' clinic of the Academic Medical Hospital of the University of Amsterdam were asked which of the 73 items had been a problem for them in the past three months. They were then asked to rate the importance of each item they had identified, using a five point Likert scale from "not very important" to "extremely important". The items chosen most often or rated most important were included in the Parkinson's disease quality of life (PDQL) questionnaire.

PHASE 2: TESTING OF THE PARKINSON'S DISEASE QUALITY OF LIFE (PDQL) QUESTIONNAIRE

Study sample

Between October 1994 and December 1994 we conducted a survey of persons with Parkinson's disease who belonged to the Dutch Parkinson's Disease Society. From the organisation's mailing list we identified 529 members by systematic sampling of zip codes (every eighth member on the list). We sent self report questionnaires to these 529 patients. In all, 450 questionnaires were returned $(85 \cdot 1 \%)$. Of these, $66(14.6 \%)$ were not completed, mainly because the member was deceased $(7 \cdot 5 \%$ of the returned questionnaires), because the patient was physically unable $(2 \%)$, or the patient was cognitively impaired $(0.9 \%)$. Consequently, 384 questionnaires $(85.3 \%$ of the returned, $72.6 \%$ of the total) could be used for data analysis.

\section{Measurements}

Standard demographic questions were used to assess sex, age, and other background variables. Factors related to parkinsonism and reported at the time of the survey included duration of disease (years since diagnosis); severity of disease as measured by the Schwab and England activities of daily living scale (10 categories) ${ }^{15}$; and medication.

To assess daily physical, emotional, and social functioning the medical outcome studies-24 (MOS-24), a "generic" (disease nonspecific) quality of life instrument, was used. The MOS-24 is a standardised measure of quality of life and is widely used for medical patients. ${ }^{1016-18}$ Seven subscales cluster 24 items under physical functioning, role functioning, social functioning, mental health, health perceptions, physical pain, and energy. The MOS-24 was scored on a five point scale. The internal consistency coefficients of the MOS24 subscales ranged from 0.69 to 0.83 .

Assessment of depression was obtained with the Center for Epidemiologic Studies depression (CES-D) scale. ${ }^{19}$ It is a short self reporting scale (20 items) and has a five-point response scale. The internal consistency coefficient of the CES-D was $0 \cdot 89$.

Social support was measured with the MOS social support survey. ${ }^{2021}$ The scales can be combined in one overall support measure. The survey has five-point Likert response scales. The internal-consistency coefficients of the MOS social support subscales ranged from 0.71 to 0.91 .

\section{Internal-consistency realibility and validity}

All data were checked for accuracy and normality distribution, and analysed using the statistical package for the social sciences (SPSSPC, 5.0). ${ }^{22}$ Reliability of the PDQL was tested by investigating the consistency of results with Cronbach's $\alpha$ coefficient. ${ }^{23}$ As recommended by Nunnally, internal estimates of a magnitude of 0.70 or greater were considered reasonable, over 0.80 as good, and over 0.90 as excellent. ${ }^{24}$

Validation of a questionnaire is a quantitative assessment of how well it measures what it claims to measure. Two approaches were taken to evaluate the validity of the PDQL.

Discriminant validity of the PDQL - that is, the extent to which the questionnaire scores were able to discriminate between subgroups of patients differing in clinical state, ${ }^{25}$ was assessed by the method of known groups comparison. One way analysis of variance (ANOVA) was employed to test for the statistical significance of differences in PDQL scores between different categories of the Schwab and England categories. To assure sufficient numbers of patients in each group, the 10 groups of the Schwab and England activities of daily living scale were combined into three new categories of severity of disease. Groups $100 \%, 90 \%$, and $80 \%$ were combined as group 1 (being independent, activities a bit slower); groups $70 \%$ and $60 \%$ were combined as group 2 (being slightly dependent, activities considerably slower); and groups $50 \%, 40 \%$, $30 \%, 20 \%$, and $10 \%$ were combined as group 3 (being dependent, needing help or care). Group 1 consisted of 171 patients, group 2 of 127 patients, and group 3 of 82 patients. It was hypothesised that severity of disease would clearly discriminate between the parkinsonian and systemic scores, but less clearly between the emotional and social scores of the PDQL. In the case of a significant effect, the Scheffé test for multiple comparison was performed to test for differences between the different Schwab and England groups.

To evaluate the convergent validity-that is, the extent to which the questionnaire is able to measure what it claims to measure, productmoment correction coefficients were calculated to assess linear relations between the PDQL and the three generic indices of quality of life. It was expected that conceptually related scales would correlate substantially (Pearson's $r>0 \cdot 40$ ).

\section{Results}

PHASE 1: ITEM SELECTION AND REDUCTION

The item reduction questionnaire was completed by 13 consecutive patients: four admitted patients and nine outpatients (six women and seven men; mean age 61.31 years).

Table 1 presents the highest scoring items for 
Table 1 Highest scoring items (37) of the item reduction questionnaire as evaluated by 13 patients

\begin{tabular}{|c|c|}
\hline Subscale of Parkinson's disease questionnaire & Overall importance * \\
\hline \multicolumn{2}{|l|}{ Parkinson symptoms: } \\
\hline Difficulties writing & 34 \\
\hline Difficulties turning around in bed & 32 \\
\hline Shaking of the hand(s) & 31 \\
\hline Shuffling & 31 \\
\hline Being tense & 31 \\
\hline Clumsiness & 29 \\
\hline Difficulties getting up from a chair & 28 \\
\hline Stiffness & 28 \\
\hline “'On/off” periods & 27 \\
\hline Difficulties turning while walking & 25 \\
\hline Difficulties sitting still (for long periods) & 24 \\
\hline Difficulties talking & 19 \\
\hline Sudden extreme movements & 16 \\
\hline Drooling & 14 \\
\hline \multicolumn{2}{|l|}{ Systemic symptoms: } \\
\hline Difficulties walking & 35 \\
\hline Feeling generally unwell & 30 \\
\hline Feeling worn out or having no energy & 24 \\
\hline Difficulties getting a good night's rest & 21 \\
\hline A feeling of extreme exhaustion & 21 \\
\hline Constipation & 18 \\
\hline Often needing to urinate and/or wetting yourself & 14 \\
\hline \multicolumn{2}{|l|}{ Emotional function: } \\
\hline Feeling insecure of yourself due to physical limitations & 28 \\
\hline Difficulties with your concentration & 26 \\
\hline Difficulties with your memory & 25 \\
\hline Feeling embarrassed because of your disease & 24 \\
\hline Being afraid of possible progressing of the illness & 24 \\
\hline Difficulties accepting your disease & 23 \\
\hline Feeling insecure of yourself around others & 22 \\
\hline Feeling worried about (the possible consequences of) surgery & 16 \\
\hline Feeling depressed or discouraged & 15 \\
\hline \multicolumn{2}{|l|}{ Social function: } \\
\hline No longer able to do your hobbies & 27 \\
\hline That your illness inhibits your sex life & 25 \\
\hline Difficulties in doing leisure or sport activities & 24 \\
\hline Being less able to go on holiday than before your illness & 24 \\
\hline Difficulties signing your name in public & 23 \\
\hline Difficulties with transport & 20 \\
\hline Having to cancel or postpone social activities & 19 \\
\hline
\end{tabular}

${ }^{\star}$ Frequency $\times$ mean importance (maximum: $13 \times 5=65$ ).

Table 2 Patient characteristics $(n=384)$

\begin{tabular}{lc}
\hline & $N o(\%)$ \\
\hline Sex: & $202(52 \cdot 6)$ \\
Men & $182(47 \cdot 4)$ \\
Women & $67 \cdot 1(\mathrm{SD} 10 \cdot 8)$ \\
Mean age (y) & $270(70 \cdot 3)$ \\
Marital status: & $114(29 \cdot 7)$ \\
Married & $63(16 \cdot 4)$ \\
Widowed/unmarried/divorced & $216(56 \cdot 3)$ \\
Occupational state: & $105(27 \cdot 3)$ \\
Unfit to work & $7 \cdot 87(\mathrm{SD} 6 \cdot 34)$ \\
Pension & \\
Other (for example, work) & $171(44 \cdot 5)$ \\
Duration of disease (y) & $127(33 \cdot 1)$ \\
Schwab and England: & $82(21 \cdot 4)$ \\
Group 1 & \\
Group 2 & $333(87 \cdot 0)$ \\
Group 3 & $54(14 \cdot 1)$ \\
Medication: & $189(49 \cdot 2)$ \\
Levodopa (with peripheral decarboxylase inhibitor) & $71(18 \cdot 5)$ \\
Bromocriptine & $21(5 \cdot 5)$ \\
Selegiline & $34(8 \cdot 9)$ \\
Amantadine & Benzodiazepines
\end{tabular}

Table 3 Descriptive statistics and reliability estimates for the PDQL $(n=384)$

\begin{tabular}{lcccc}
\hline Measure & $\begin{array}{l}\text { Scale } \\
\text { mean }(S D)\end{array}$ & $\begin{array}{l}\text { Item } \\
\text { mean }\end{array}$ & $\begin{array}{l}\text { No of } \\
\text { items }\end{array}$ & $\begin{array}{l}\text { Internal } \\
\text { consistency }\end{array}$ \\
\hline PDQL total score & $122 \cdot 9(26 \cdot 7)$ & $3 \cdot 32$ & 37 & $0 \cdot 94$ \\
$\quad$ Parkinsonian symptoms & $43 \cdot 8(10 \cdot 7)$ & $3 \cdot 12$ & 14 & $0 \cdot 86$ \\
Systemic symptoms & $22 \cdot 9(6 \cdot 2)$ & $3 \cdot 29$ & 7 & $0 \cdot 80$ \\
Emotional functioning & $31.5(7 \cdot 5)$ & $3 \cdot 50$ & 9 & $0 \cdot 87$ \\
Social functioning & $24 \cdot 2(6.9)$ & 3.46 & 7 & $0 \cdot 82$ \\
\hline
\end{tabular}

Internal consistency $=$ Cronbach's internal consistency reliability coefficient $\alpha$; higher scores represent better quality of life.

all patients on each subscale. The score opposite each item represents the product of the number of people selecting the item and the mean importance they attributed to it (frequency-importance product, range 0-65). To keep the questionnaire short, only items with a score higher than 13 on the item reduction questionnaire were selected for the PDQL. The PDQL consists of 37 items. In selecting time specification we used three months, because of the chronic nature of Parkinson's disease. In selecting response options we chose a five-point Likert scale, because of its reliability. ${ }^{26}$

The questionnaire is analysed directly from the scores recorded. The mean scores for the items within each subscale are calculated for each patient. The overall quality of life score can be calculated from the mean score over all items. (See appendix for the translated PDQL questionnaire.)

PHASE 2: TESTING OF THE QUESTIONNAIRE The mean age of the patients in the study was $67 \cdot 1$ (range 29-100) years. Women comprised $47 \%$ of the sample. Table 2 presents patient characteristics.

\section{Descriptive statistics and scale reliability}

On the basis of factor analysis, items of the PDQL have been placed in four subscales: parkinsonian symptoms, systemic symptoms, emotional functioning, and social functioning. Some items (1, 4, 5, 30, and 37) had equal factor loadings on two or more factors. In this case, items have been placed in a subscale on the basis of face validity. The PDQL and the four subscales had a normal distribution and a normal spread. Table 3 gives descriptive statistics for the four PDQL subscales and the overall PDQL scale of the 384 patients and internal consistency reliability coefficients. The quality of life scores were somewhat lower for the parkinsonian symptoms and systemic symptoms compared with the emotional and social functioning subscales $(3.12$ and $3.29 v$ 3.50 and 3.46 respectively). Score distributions on all subscales were roughly symmetric.

Internal-consistency reliability estimates were high for the PDQL subscales $(0 \cdot 80-0 \cdot 87)$ and for the PDQL overall score $(0.94)$, all exceeding the $0 \cdot 70$ standard. ${ }^{24}$

Discriminant validity: known groups comparisons. The figure gives the item mean scores of the four PDQL subscales. As expected, all scores were systematically lower (indicating poorer quality of life) in groups with higher disease severity $(P<0.001)$. For the overall PDQL, patients in group 1 (independent, activities a bit slower) had a mean score of 137 , patients in group 2 (slightly dependent, activities considerably slower) had a mean score of 118 , and patients in group 3 (dependent, needing help or care) had a mean score of 98 .

The scores of the parkinsonian, systemic, emotional, and social function subscales followed a similar pattern. As expected, patients with higher disease severity had lower scores on the Parkinson $(\mathrm{P}<0.001)$ and the systemic scale $(\mathbf{P}<0.001)$. Moreover, they also had lower scores on the emotional $(P<0.001)$ and the social scales $(P<0.001)$. The scores of each of the three groups were significantly different from the scores of the other two groups on all four subscales and on the total PDQL scale (Scheffé procedure, all possible pairs of groups $\mathrm{P}<0.05)$. 


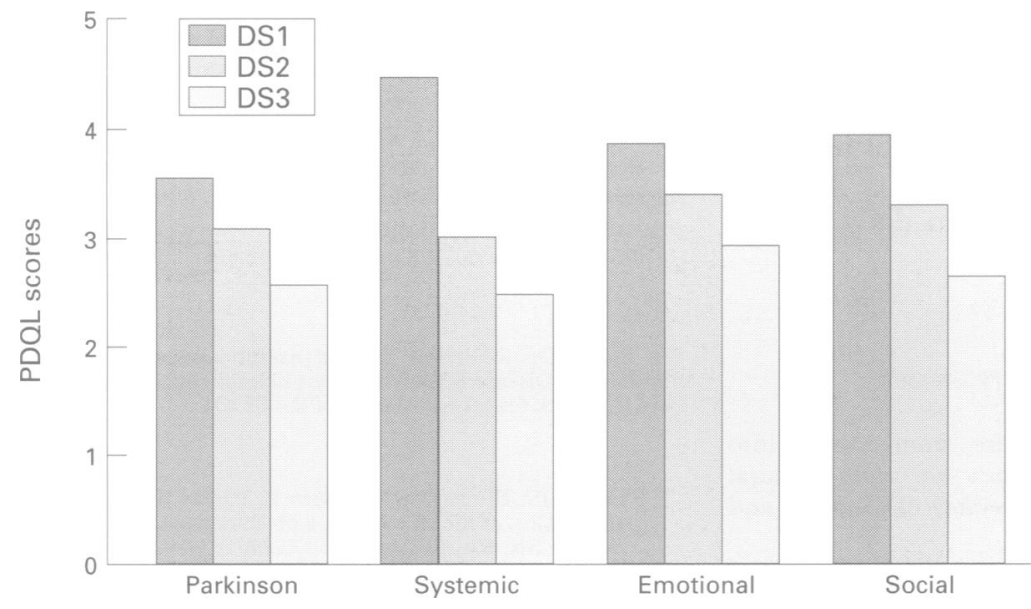

$P D Q L$ scores by disease severity (DS). ANOVAs comparing mean PDQL scores for patients in the three disease severity (DS) groups: $P<0.001$ for all $P D Q L$ scores. Higher scores represent better quality of life. Completely dependent (DS1, $n=171$ ); somewhat dependent (DS2, $n=127)$; dependent (DS3, $n=82)$.

Table 4 Correlation matrix of $P D Q L$ and generic indices of quality of life

\begin{tabular}{lcccc}
\hline & \multicolumn{2}{l}{$P D Q L$ measure } & & \\
\cline { 2 - 4 } Generic quality of life indices & Parkinsonian & Systemic & Social & Emotional \\
\hline Physical functioning (+) & 0.50 & 0.48 & & \\
Role functioning (+) & 0.48 & 0.46 & & \\
Health perceptions (+) & 0.46 & 0.52 & & \\
Energy/fatigue (+) & 0.50 & 0.64 & & \\
Bodily pain (-) & -0.22 & -0.36 & 0.43 & \\
Social functioning (+) & & & 0.13 & 0.66 \\
Social Support (+) & & & & -0.79 \\
Mental health (+) & & & & \\
Depression CES-D (-) & & & & \\
\hline
\end{tabular}

Values are Pearson correlation coefficients $(r)$; values $>0.19$ are statistically significant $(P<$ $0.001) ;+/-$ in parentheses $=$ direction of scoring (a negative sign indicates poorer health or functioning, a positive sign indicates better health or functioning).

Convergent validity

Table 4 shows the matrices of productmoment correlation coefficients for PDQL subscale scores and three generic indices of quality of life. It was expected that conceptually related scales would correlate substantially. All correlations were statistically significant $(P<0.01)$. The correlations between the PDQL scores and the generic quality of life indices (the MOS-24 subscales, the social support survey, and the CES-D depression scale) exceeded the 0.40 criterion for conceptually related scales for seven of the nine hypothesised subscales. High correlations were found between the physical subscales and the parkinsonian and systemic symptoms, except for the correlations with physical pain. Highest correlations were between the emotional function scale of the PDQL and both the mental health subscale of the MOS and the depression scale. The social function subscale had high correlations with the social functioning scales of the MOS, but not with the social support survey.

\section{Discussion}

We have developed a quality of life instrument for patients with Parkinson's disease which examines parkinsonian symptoms, systemic symptoms, emotional functioning and social functioning. The PDQL has several advan- tages over existing measurements of functional state. Items were generated from statements about disease related problems of daily living made by patients and neurologists and from a review of the medical literature. Other patients identified which items of impairment of their quality of life were most important to them. These items were incorporated in the questionnaire. This selection process ensures that questionnaire items concentrate on areas of dysfunction most important to patients with Parkinson's disease. The questionnaire is given directly to the patients. Whereas existing disease specific measures focus on tremor or rigidity, our questionnaire also includes other major aspects of dysfunction. The highest scores were in items relating to parkinsonian symptoms, systemic symptoms, and some aspects of emotional function. On average, social problems were scored as less prevalent and important.

The questionnaire is appropriate for most patients with Parkinson's disease, but cannot be given to those with significant cognitive impairment (less than $1 \%$ in our sample). We found that patients with Parkinson's disease took about twice as long to fill in the same questionnaires as patients with inflammatory bowel disease. ${ }^{27}$ Nevertheless, most patients $(85 \%)$ were willing to participate. Most respondents $(69.9 \%)$ completed the questionnaire by themselves.

Internal consistency (reliability) of the questionnaire was well above accepted levels. The questionnaire differentiated across three clinical groups as predicted. Mobile and independent patients have better quality of life scores than slightly dependent patients and slightly dependent patients have better quality of life scores than dependent patients who need help or care. Whether it can also discriminate between normal subjects and those with Parkinson's disease is not relevant as this is not the purpose of the instrument. Severity of disease, however, was scored by the patient and not by the neurologist. It is therefore possible that the patient's score on the Schwab and England scale reflected the patient's quality of life rather than disease severity, although the wording of the Schwab and England questionnaire focused solely on the activities of daily living and did not refer to quality of life.

As expected, the PDQL correlated with established generic measures of quality of life: the MOS-24 quality of life scale, and the CES$\mathrm{D}$ depression scale. It can be assumed that they reflect similar constructs and domains of quality of life. Therefore, the PDQL is a valid instrument.

The results reported here require confirmation by other investigators. More research has to be done to see how the PDQL will behave longitudinally. The PDQL takes only a few minutes to administer. We therefore recommend that investigators interested in determining the effects of their interventions on quality of life in patients with Parkinson's disease should give the PDQL as a disease specific questionnaire. As a time specification we used three months, because of the chronic 
nature of Parkinson's disease, but this could be modified according to the study.

In conclusion, the PDQL is an appropriate, validated and potentially useful tool to assess quality of life in clinical studies of patients with Parkinson's disease and in daily clinical practice.

\section{Appendix}

THE PARKINSON'S DISEASE QUALITY OF LIFE

QUESTIONNAIRE (PDQL)

We translated the questionnaire from Dutch into English, following the guidelines for cross cultura adaptation of quality of life measures of Guyatt ${ }^{28}$ and Guillemin et al. ${ }^{29}$

To obtain semantic, idiomatic, and conceptual equivalence in translation we used back translation techniques. This involved using native bilingual English and Dutch speakers who translated the questionnaire into their mother tongue. A small committee of bilingual members compared source and back translation versions and produced a final version as follows:

How often during the last three months did you have trouble with:

Response options:

1) All of the time

2) Most of the time

3) Some of the time

4) A little of the time

5) Never

1. stiffness $(\mathbf{P})$

2. feeling generally unwell (Sys)

3. that you are no longer able to do your hobbies (Soc)

4. being tense $(P)$

5. feeling insecure of yourself due to your physical limitations (E)

6. shaking of the hand(s) (P)

7. feeling worn out or having no energy (Sys)

8. difficulties in doing sport or leisure activities (Soc)

9. clumsiness $(\mathrm{P})$

10. feeling embarrassed because of your illness $(E)$

11. shuffling $(\mathrm{P})$

12. having to postpone or cancel social activities because of your illness (Soc)

13. a feeling of extreme exhaustion (Sys)

14. difficulties turning around while walking $(P)$

15. being afraid of possible progressing of the illness (E)

16. difficulties writing $(P)$

17. being less able to go on holiday than before your illness (Soc)

18. feeling insecure of yourself around others (E)

19. difficulties getting a good night's rest (Sys)

20. "on/off" periods (P)

21. difficulty with accepting your illness (E)

22. difficulties talking $(P)$

23. difficulties signing your name in public (Soc)

24. difficulties walking (Sys)

25. drooling $(\mathrm{P})$

26. feeling depressed or discouraged (E)

27. difficulty with sitting still (for long periods) (P)

28. often needing to urinate and/or wetting yourself (Sys)

29. difficulties with transport (Soc)

30. sudden extreme movements $(P)$

31. difficulties concentrating (E)

32. difficulties getting up from a chair $(P)$

33. constipation (Sys)

34. difficulties with your memory (E)

35. difficulties turning around in bed $(\mathbf{P})$

36. that your illness inhibits your sex life (Soc)

37. feeling worried about (the possible consequences of) an operation in connection with your illness (E)

(P): Parkinson symptoms

(Sys): Systemic symptoms

(Soc): Social functioning

(E): Emotional functioning
^These scales are not intended to be used without permission. Investigators interested in using the instrument should contact the first author. For academic use, permission will be granted at no charge, but while still under development, investigators will be requested to share their results with the instrument so that reliability and validity testing can proceed appropriately. Commercial institutions will be asked for a contribution for the use of the instrument.

We thank Dr $\mathrm{R}$ Brown for his useful advice; the Dutch Parkinson's Disease Society for their helpful collaboration; and Paul Bundock for his translation of the PDQL

1 Shindler JS, Brown RG, Welburn P, Parkes JD. Measuring the quality of life of patients with Parkinson's disease. In Walker SR, Rosser RM, eds. Quality of life: assessment and application. Lancaster: MTP Press Ltd, 1988

2 Starkstein SE, Mayberg HS, Leiguarda R, Preziosi TJ, Robinson RG. A prospective longitudinal study of depression, cognitive decline and physical impairment in patients with Parkinson's disease. $\mathcal{f}$ Neurol Neurosurg Psychiatry 1990;55:377-82.

3 Welburn P, Walker S. Assessment of quality of life in Parkinson's disease. In: Smith GT, ed. Measuring health: a practical approach. New York: J Wiley, 1988.

4 Hoehn MM, Yahr MD. Parkinsonism: onset, progression and mortality. Neurology 1967;17:427-42.

5 Diamond SG, Markham $\mathrm{CH}$. Evaluating the evaluations: or how to weigh the scales of parkinsonian disability. Neurology 1983;33:1098-9.

6 Mutch WJ, Smith WC, Scott RF. A screening and alerting questionnaire for parkinsonism. Neuroepidemiology 1991;

7 Lakke JPWF. Assessment and measurement. In: Stern S, ed. Parkinson's disease. London: Chapman and Hall Medical 1990.

8 Bergner M, Bobbit RA, Carter WB, Gilson BS. The sickness impact profile: development and final revision of a health status measurement. Med Care 1981;19:787-805.

9 Hunt SM, McEwan J, McKenna SP. Measuring health status. London: Croom Helm, 1986.

10 Stewart AL, Hays RD, Ware JE. The MOS short-form general health survey: reliability and validity in a patient population. Med Care 1988;26:724-35.

11 Guyatt GH, Bombardier C, Tugwell PX. Measuring disease-specific quality of life in clinical trials. Can Med Assoc $\Im 1986 ; 134: 889-95$

12 Kirshner B, Guyatt G. A methodological framework for assessing health indices. Fournal of Chronic Diseases 1985 38:27-36.

13 Guyatt GH, Mitchell A, Irvine EJ, et al. A new measure of health status for clinical trials in inflammatory bowel disease. Gastroenterology 1989;96:804-810.

14 Juniper EF, Guyatt GH, Epstein RS, Ferrie PJ, Jaeschke R Hiller TK. Evaluation of impairment of health related quality of life in asthma: development of a questionnaire for use in clinical trials. Thorax 1992;47:76-83.

15 Schwab RS, England AC. Projection technique for evaluating surgery in Parkinson's disease. In: Gillingham FJ, ing surgery in Parkinson's disease. In. Gillingham sis Donaldson MC, eds. Third symposium on

16 Stewart AL, Greenfield S, Hays RD, et al. Functional status and well-being of patients with chronic conditions. $\mathscr{A} A M A$ and well-being of

17 Stewart AL, Ware JE. Measuring functioning and well-being. Durham: Duke University Press, 1992.

18 Wells KB, Stewart AL, Hays RD, et al. The functioning and well-being of depressed patients. $¥ A M A 1989 ; 262: 914-9$.

19 Radloff LS. The CES-D scale: a self-report depression scale for research in the general population. Applied Psychological Measurement 1977;1:385-401.

20 Sherbourne CD, Stewart AL. The MOS social support survey. Soc Sci Med 1991;32:705-14.

21 Sherbourne CD, Meredith LS, Rogers W, Ware JE. Social support and stressful life events: age differences in their support and stressful life events: age differences in their cally ill. Oual Life Res 1992;1:235-46.

22 Norusis MI. SPSS-PC+ Base system user's guide, version 5.0 Chicago: SPSS Inc, 1992

23 Cronbach LJ. Coefficient Alpha and the internal structure of tests. Psychometrika 1951;16:297-334.

24 Nunnally JC. Psychometric theory, 2nd ed. New York McGraw-Hill, 1978 .

25 Crocker L, Algina J. Introduction to classical and modern test theory. New York: Holt, Rinehart and Winston, 1986.

26 McKelvie JS: Graphic rating scales: how many categories? $\mathrm{Br}$ f Psychol 1978;69:185-202.

27 De Boer AGEM, Wijker W, Bartelsman JFW, De Haes JCJM. Inflammatory bowel disease questionnaire: crosscultural adaptation and further validation. European fournal of Gastroenterology and Hepatology 1995;7: $1043-550$.

28 Guyatt GH. The philosophy of health-related quality of life translation. Quality of Life Research 1993;2:461-5.

29 Guillemin F, Bombardier C, Beaton, D. Cross-cultural adaptation of health-related quality of life measures: literature review and proposed guidelines. $\mathcal{f}$ Clin Epidemiol ture review and prot 